\title{
Community-based aftercare following an emergency department presentation for attempted suicide or high risk for suicide: study protocol for a non-randomised controlled trial
}

\author{
Vida V. Bliokas ${ }^{1,2^{*}}$ (D) Alex R. Hains ${ }^{2,3,4}$, Jonathan A. Allan ${ }^{1}$, Luise Lago ${ }^{5}$ and Rebecca Sng ${ }^{6}$
}

\begin{abstract}
Background: Suicide is a major public health issue worldwide. Those who have made a recent suicide attempt are at high risk for dying by suicide in the future, particularly during the period immediately following departure from a hospital emergency department. As such the transition from hospital-based care to the community is an important area of focus in the attempt to reduce suicide rates. There is a need for evaluation studies to test the effectiveness of interventions directed to this stage (termed 'aftercare' interventions).

Methods: A controlled non-randomised two group (intervention vs treatment-as-usual control) design, using an intention-to-treat model, will evaluate the effectiveness of a suicide prevention aftercare intervention providing follow-up after presentations to a hospital emergency department as a result of a suicide attempt or high risk for suicide. The intervention is a community-based service, utilising two meetings with a mental health clinician and follow-up contacts by peer workers via a combination of face-to-face and telephone for four weeks, with the option of extension to 12 weeks. Seventy-five participants of the intervention service will be recruited to the study and compared to 1265 treatment-as-usual controls. The primary hypotheses are that over 12 months, those who participate in the aftercare follow-up intervention are less likely than controls to present to a hospital emergency department for a repeat suicide attempt or because of high risk for suicide, will have fewer re-presentations during this period and will have lower all-cause mortality. As a secondary aim, the impact of the intervention on suicide risk factors for those who participate in the service will be evaluated using pre- and post-intervention repeated measures of depression, anxiety, stress, hopelessness, belongingness, burdensomeness, and psychological distress. Enrolments into the study commenced on 1 November 2017 and are anticipated to cease in November 2019.
\end{abstract}

Discussion: The study aims to contribute to the understanding of effective interventions for individuals who have presented to a hospital emergency department as a result of a suicide attempt or at high risk for suicide and provide evidence in relation to interventions that incorporate peer-workers.

Trial registration: ACTRN12618001701213. Registered on 16 October 2018. Retrospectively registered.

Keywords: Suicide prevention, Aftercare, Peer worker

\footnotetext{
* Correspondence: vida@uow.edu.au

${ }^{1}$ University of Wollongong, Northfields Avenue, Wollongong, NSW 2522,

Australia

${ }^{2}$ Illawarra Health and Medical Research Institute, Northfields Avenue,

Wollongong, NSW 2522, Australia

Full list of author information is available at the end of the article
}

(c) The Author(s). 2019 Open Access This article is distributed under the terms of the Creative Commons Attribution 4.0 International License (http://creativecommons.org/licenses/by/4.0/), which permits unrestricted use, distribution, and

reproduction in any medium, provided you give appropriate credit to the original author(s) and the source, provide a link to the Creative Commons license, and indicate if changes were made. The Creative Commons Public Domain Dedication waiver (http://creativecommons.org/publicdomain/zero/1.0/) applies to the data made available in this article, unless otherwise stated. 


\section{Background}

Suicide is a major public health issue worldwide [1]. In Australia, 3128 people died by suicide in 2017 (12.7 per $100,000)$, with suicide the leading cause of death amongst 15-44 year olds [2]. When it comes to suicidal behaviours more broadly, deaths are considered 'the tip of the iceberg' [3], with estimates of approximately 30 suicide attempts for every one suicide death [4]. Those who have made a recent suicide attempt are at high risk for dying by suicide in the future $[5,6]$, particularly immediately after discharge from a hospital emergency department [7] and within the first year following a suicide attempt $[5,8]$.

The most common point of contact that people who have attempted suicide have with support services is via a hospital emergency department [9]. However, people who have presented to an emergency department following a suicide attempt have described the support received as either insufficient [9] or completely absent [10-13]. As a result, there have been numerous calls to improve the support people receive within emergency departments and improve the way they are connected with ongoing community-based care $[14,15]$.

In the context of physical health care, the quality of the discharge planning and transfer of care is known to have significant impacts on patient outcomes [16-21]. Therefore, improved transition from hospital-based care to community supports for people presenting to an emergency department following a suicide attempt is a priority for effective suicide prevention [14, 15]. This type of intervention is referred to as 'aftercare'.

Aftercare is consistently included as a key component of multilevel initiatives, or a 'systems approach' to suicide prevention [22-26]. One such systems approach is the LifeSpan project [23], which is being coordinated by the Black Dog Institute and implemented within four regions of New South Wales, Australia, including the Illawarra Shoalhaven region. The LifeSpan project involves implementing nine suicide prevention strategies simultaneously. Of all these strategies, implementing effective aftercare is estimated to reduce suicide attempts by $19.8 \%$, the biggest impact from any one strategy in the LifeSpan systems approach [27].

The first landmark study evaluating the impact of a coordinated aftercare service was conducted on the outskirts of Oslo, Norway [28]. In what became known as 'the Bærum Model', people who presented to hospital after attempting suicide (but were not directly admitted) were followed up with home visits for up to 6 months and provided with support via a combination of face-toface and phone contacts. There were four components to the support offered: (1) outreach - immediate, assertive outreach and follow-up after discharge from hospital, (2) problem-solving - solution focused counselling, (3) adherence - encouragement to remain engaged with treatment and follow recommendations, and (4) continuity - maintain contact with a consistent group of support people [29]. This intervention was credited with having contributed to a significant reduction in suicidal behaviours, from 170 per 100,000 in 1984 to 79 per 100 , 000 in 1995; a reduction of 53.5\% [28].

These findings were supported by a Danish randomised controlled trial of a 6-month aftercare service based on the Bærum Model [30], in which 69 intervention participants were compared with 64 treatment-asusual controls. The researchers found that significantly fewer people in the intervention group re-attempted suicide $(8.7 \%)$ than the control group $(21.9 \%)$ over a 12 month period. This study also found reduced numbers of repeated acts ( 8 in the intervention group versus 22 in the control group). The researchers noted that a key characteristic of the intervention was its "swift and rather aggressive outreach" [30] p.296, as well as the capacity to adapt the support to account for personal factors. A 5-year follow-up study found the number of people with repeated suicide attempts remained lower for the intervention group for up to 3-4 years, and the total number of repeat suicide attempts remained lower for the intervention group after 5 years [31].

Fundamentally, aftercare interventions aim to bridge the transition between an emergency department and community-based supports, with the key factor being a focus on making and keeping contact to ensure a constant sense of connectedness [31, 32]. As such, aftercare can be delivered via a number of methods - face-to-face contact [29], phone contact only [33, 34], a combination of phone and face-to-face [35], or mailed out letters [36-39]. The aftercare models evaluated have also ranged in duration, from a single contact [40] to regular contacts over 18 months [35]. Each of these models have illustrated the potential for aftercare services to have an impact on suicidal behaviours for those who have attempted suicide.

\section{Peer workers}

Aftercare services have also varied in terms of their staffing profile, typically using a combination of doctors, nurses, psychologists, community workers, and social workers [41]. However, there are no records in the literature of aftercare services utilising peer workers; people employed on the basis of their personal lived experience of suicide or mental illness and recovery [42]. There is a growing body of evidence to support the use of peer workers in assisting people who are experiencing mental illness [42]. A review of four randomised controlled trials by Davidson, Chinman, Sells and Rowe [43] found that, across most outcomes, peer workers were just as effective as professionals in providing case management 
and support for individuals with severe mental illness. Furthermore, one of the studies reviewed found significantly fewer hospitalisations for those who were being case managed by peer workers compared to those being managed by a mental health professional [44]. Similarly, a reduction in the number of hospital re-admissions over a three-year period for mental health patients who received peer support has been observed [45]. These findings suggest there are potential benefits to involving peer workers in mental health care, although thus far, the majority of studies have been qualitative in nature and the few randomised controlled trials that have been conducted have primarily focussed on case management interventions $[43,46,47]$. A literature search conducted by the authors did not produce any studies that had specifically focussed on the use of peer workers with suicidal clients or in the context of aftercare services. As a result, there is a need for research to explore the effectiveness of a suicide prevention intervention utilising peer workers.

\section{GROW coaching model}

In addition to providing a bridge between crisis (hospital-based) care and ongoing (community-based) care, the Bærum aftercare model also emphasised the importance of a practical problem-solving approach. An example of such an approach is provided by the GROW coaching model (Goal, Reality, Options, Wrap-up). The GROW model is a simple, collaborative, solution focussed framework that informs the structure of intervention sessions with clients [48-50]. Clients are firstly asked to set a goal (G) for what they would like to achieve during the coaching session. They are encouraged to create a goal that is specific and within their control, and they are assisted in breaking down larger goals into smaller, concrete steps [51]. Secondly, clients are assisted in gaining a better understanding of their reality (R), or current situation $[49,50]$. They are encouraged to consider what they have previously found helpful, what they have found to be unhelpful, and any barriers they may be experiencing regarding their goals [51]. Thirdly, clients are encouraged to evaluate different options $(\mathrm{O})$ for overcoming likely barriers to achieving goals $[48,51]$. The fourth stage of the GROW model is the wrap-up (W) $[49,50]$, which encourages clients to create specific action steps in order to move forward, overcome barriers and evaluate their progress [48]. An advantage of a practical problem-solving framework, such as the GROW model is that it can be implemented, with relatively brief training, by staff of any background, including non-professionals.

\section{Targeting suicidal thoughts and behaviours}

A critical factor in suicide prevention interventions is to directly target suicidal thoughts and behaviours [52]. An empirically supported example is the Collaborative Assessment and Management of Suicide (CAMS) model $[53,54]$, in which a clinician and client work collaboratively through goal-setting, treatment planning and monitoring [53]. Clients are asked to; outline the key problems or 'drivers' that are contributing to their suicidality and then work alongside a clinician to produce a set of goals and objectives to alleviate these problems; consider alternative behaviours to self-harm that they can engage in if in a suicidal crisis (e.g., going for a walk; calling an ambulance; people they can contact for help); and rate their suicide risk factors. Clients evaluate their goals and stabilisation plan at every treatment appointment $[53,54]$. The structure of the CAMS framework is maintained within treatment sessions via the application of the Suicide Status Form (SSF), which is a tool that guides the conversation and collaborative work between the clinician and client.

Application of the CAMS framework has been shown to reduce suicidal ideation and/or behaviours amongst college students [54,55] and defence personnel [56], as well as both outpatients $[57,58]$ and inpatients [59-61]. It has been found to be effective when adapted to online learning modalities [62] and has also demonstrated efficacy when delivered by clinicians from a range of disciplines [63]. The CAMS framework has not, to the authors' knowledge, been applied within an aftercare context.

This paper describes the study protocol for a nonrandomised controlled trial of an aftercare suicide prevention intervention that will provide follow-up to people who have presented to a hospital emergency department following a suicide attempt or with significant suicide risk, and who are not admitted to hospital as an inpatient, or being actively case managed by mental health services, at that time. The intervention, known in the region as 'Aftercare', will utilise clinicians and peer workers, and provide practical solution-focused intervention and targeted intervention of suicidal thoughts and behaviours. The Aftercare intervention will utilise the GROW coaching model and apply the SSF from the CAMS framework to target suicidal thoughts and behaviours directly. The intervention will involve a combination of face-to-face and telephone sessions over a fourweek period, with the option of extension to 12 weeks.

\section{Methods/design \\ Study aims}

The primary objectives of the study are to evaluate the impact of the Aftercare intervention on the likelihood of a re-presentation to an emergency department as a result of a suicide attempt or because of high risk for suicide, the rates of re-presentation to emergency departments as a result of one or more repeat suicide attempt(s) or because of high risk for suicide, and the 
number of deaths by any cause. The secondary objectives of the study are to evaluate the impact of the Aftercare intervention on factors associated with increased risk of suicide, namely, mood [64], hopelessness [65, 66], belongingness, burdensomeness [66], and psychological distress $[67,68]$.

It is hypothesised that;

1) Participants of the Aftercare intervention will be less likely to have a repeat presentation to an emergency department within 12 months as a result of a suicide attempt or being at high risk for suicide than non-participants.

2) Participants of the Aftercare intervention will have lower rates of re-presentations to a hospital emergency department for suicide attempt or high risk for suicide within 12 months than non-participants.

3) Participation in the Aftercare intervention will be associated with fewer all-cause deaths within 12 months than non-participation.

4) For participants of the Aftercare intervention, depression, anxiety, stress, hopelessness, belongingness, burdensomeness, and psychological distress will be improved in comparison to baseline levels following the intervention.

\section{Study setting}

The Aftercare intervention will be implemented in the Illawarra Shoalhaven region on the south-east coast of New South Wales (NSW), Australia. The region has a population of just over 400,000 and is served by three main public hospitals and several smaller regional hospitals [69]. The region experiences a suicide rate of 12.6 per 100,000, which is above the NSW state average of 10.6 per 100,000 [2], and more than 1000 suicide-related presentations to emergency departments each year [70].

The intervention will be implemented by a collaboration of three community-based organisations - Grand Pacific Health, Flourish Australia, and South Coast Medical Service Aboriginal Corporation. Referrals to the Aftercare service will be received by the service providers from the emergency departments of the three main hospitals.

\section{Study design}

A controlled non-randomised two group (intervention vs treatment-as-usual (TAU) control) design will be used to test the primary hypotheses (hypotheses 1-3), following the intention-to-treat method. The secondary objectives of the study will be evaluated using a pre- and post-intervention repeated measures design (hypothesis 4), in which the intervention group (i.e. participants of the Aftercare intervention) will complete self-report questionnaires prior to their initial intervention session, and again at the time of their discharge appointment from the service.

\section{Study duration}

The Aftercare intervention commenced receiving referrals on 21 August 2017 from the emergency department of the largest hospital in the region, the Wollongong Hospital, and following the receipt of human research ethics approval, recruitment of study participants commenced on 1 November 2017. The service was rolled out gradually, so the full implementation of the intervention, including referrals from all three participating emergency departments, occurred on 5 March 2018. Data will continue to be collected for 12 months following the recruitment of the 75th intervention group study participant. As such, data collection for the study is forecast to cease by November 2020 .

\section{Study sample}

Participants in the Aftercare intervention will be drawn from people who attend one of the three participating emergency departments as a result of a suicide attempt or high risk for suicide, who meet the referral inclusion criteria. Participants' risk for suicide (and therefore suitability for the Aftercare intervention) will be determined by the hospital staff using standard mental health assessment protocols. Those considered for referral to the Aftercare intervention will be 16 years of age and over. Those excluded from consideration for the service will be admitted to the hospital as an inpatient, or being actively case managed by mental health services, at that time of presentation to the emergency department. Those who consent to participate in the Aftercare intervention and who consent for their data to be included in the study will form the intervention group for the study.

The control group (TAU) will be sourced via deidentified hospital data extraction. They will be people 16 years of age and over who attend a participating emergency department as a result of a suicide attempt or high risk for suicide but either do not receive, or do not accept, a referral to the Aftercare intervention (i.e. did not wish to participate in the service; were unable to participate for other reasons e.g., time commitments; or had not received a referral to the service e.g. standard procedures were not followed in the emergency department). Pre-determined hospital codes for suicide attempt or high risk for suicide will identify controls. They will be selected by identifying those patients who presented to any of the emergency departments in the study during the study period, and identifying those who presented in the same month, who met the study inclusion criteria, and who had the closest emergency department presentation dates to the participants undertaking the Aftercare intervention and enrolled in the trial. A human 
research ethics waiver for consent was granted; all data is sourced via hospital data and code sets.

\section{Inclusion and exclusion criteria}

Health consumers who are aged 16 years of age and over who present to an emergency department with significant suicidality will be considered for the Aftercare intervention. They will be ineligible to participate if they are admitted to the hospital as an inpatient, if they are being actively case managed by the local health district's mental health services, or if they have cognitive impairment sufficient to preclude full participation in, or comprehension of, the intervention as assessed by the referring clinician.

\section{Study procedure}

Health consumers attending their local emergency department following a suicide attempt or at high risk for suicide are provided with a mental health assessment by clinical staff, where risk for suicide (and therefore suitability for the Aftercare intervention) is determined by standard hospital mental health assessment protocols. Staff determine whether the consumer warrants admission to hospital, as per the NSW Ministry of Health policies. If the consumer is not admitted to hospital as an inpatient, standard procedures include one follow-up phone call or home visit by the local health district's mental health outreach team within seven days and a discharge letter to the person's general practitioner. If the consumer is eligible for referral to the Aftercare service he/she will also be given a brief overview of the service by an emergency department clinician and at that time may choose to provide verbal consent to be referred to the service. The emergency department staff will contact the Aftercare service by phone and provide a referral. The participant flow process is shown in Fig. 1.

\section{Aftercare intervention}

The Aftercare service is administered through a collaboration of three community-based organisations in the region; Grand Pacific Health, Flourish Australia and the South Coast Medical Service Aboriginal Corporation. Referrals are taken by Grand Pacific Health and allocated according to location.

The consumer will be contacted by an Aftercare peer worker within one hour of the referral being received from the emergency department, while the consumer is still in the emergency department; or if the referral is made outside of operational hours they will be contacted the following day. A brief overview of the Aftercare program will be provided and, with consent, an initial community-based appointment with an Aftercare mental health clinician will be scheduled for the following day, or otherwise, as best convenient for the participant within the shortest possible timeframe.

Participants of the Aftercare service will attend the community-based service for their first appointment. Prior to their appointment, they will be provided with the research participant information sheet and consent form, and will have the opportunity to discuss the research further. Following this, they will complete the pre-intervention measures via tablet computer (or using pen and paper when appropriate).

The initial appointment with a mental health clinician will involve a collaborative assessment and planning session and take approximately one hour. The assigned peer worker will also be present and, with the consent of the participant, the participant's family/carer will be encouraged to attend. Participants will complete the Suicide Status Form-4 (SSF-4) [53] with the mental health clinician. The SSF-4 is a multi-component clinical semistructured assessment and treatment planning tool designed to assist in assessing the level of suicide risk and factors associated with suicidal thoughts. Six five-point Likert scales rate the degree of psychological pain, stress, agitation, self-hopelessness, self-hate, and overall suicide risk; and additional open-ended questions ask participants to elaborate upon these factors (e.g., "What I find most painful is..."). An earlier version of the form has demonstrated good convergent validity of these rating items with other established measures [55].

In addition, the SSF-4 has items which rate the degree to which participants attribute their suicidal thoughts and feelings to themselves (internal) or others (external), as well as their desire to live and their desire to die. Participants are also asked to make a list of their reasons for living versus their reasons for dying. The form then directs the interaction towards seeking solutions, via the question "the one thing that would make me no longer suicidal would be?" before the clinician gathers information regarding 14 risk factors for suicide (e.g. access to means, history of suicide attempts, substance use). The participant and clinician will document on the SSF-4 the agreed aftercare and stabilisation plans (e.g., "Things I can do to cope differently when I am in a suicide crisis"). The SSF-4 will be repeated at each contact with the clinician.

In the aftercare plan, participants will be asked to set personal goals, consider the values underpinning the goals, short-term and long-term action plans, their strengths and resources, and how to measure successful goal achievement. The aftercare plan, along with the stabilisation plan, will form the basis for individualised ongoing support, and will also be shared with the participant's nominated general medical practitioner and other relevant stakeholders, with permission. Should family or other support people nominated by the participant attend the session, they will be provided with 


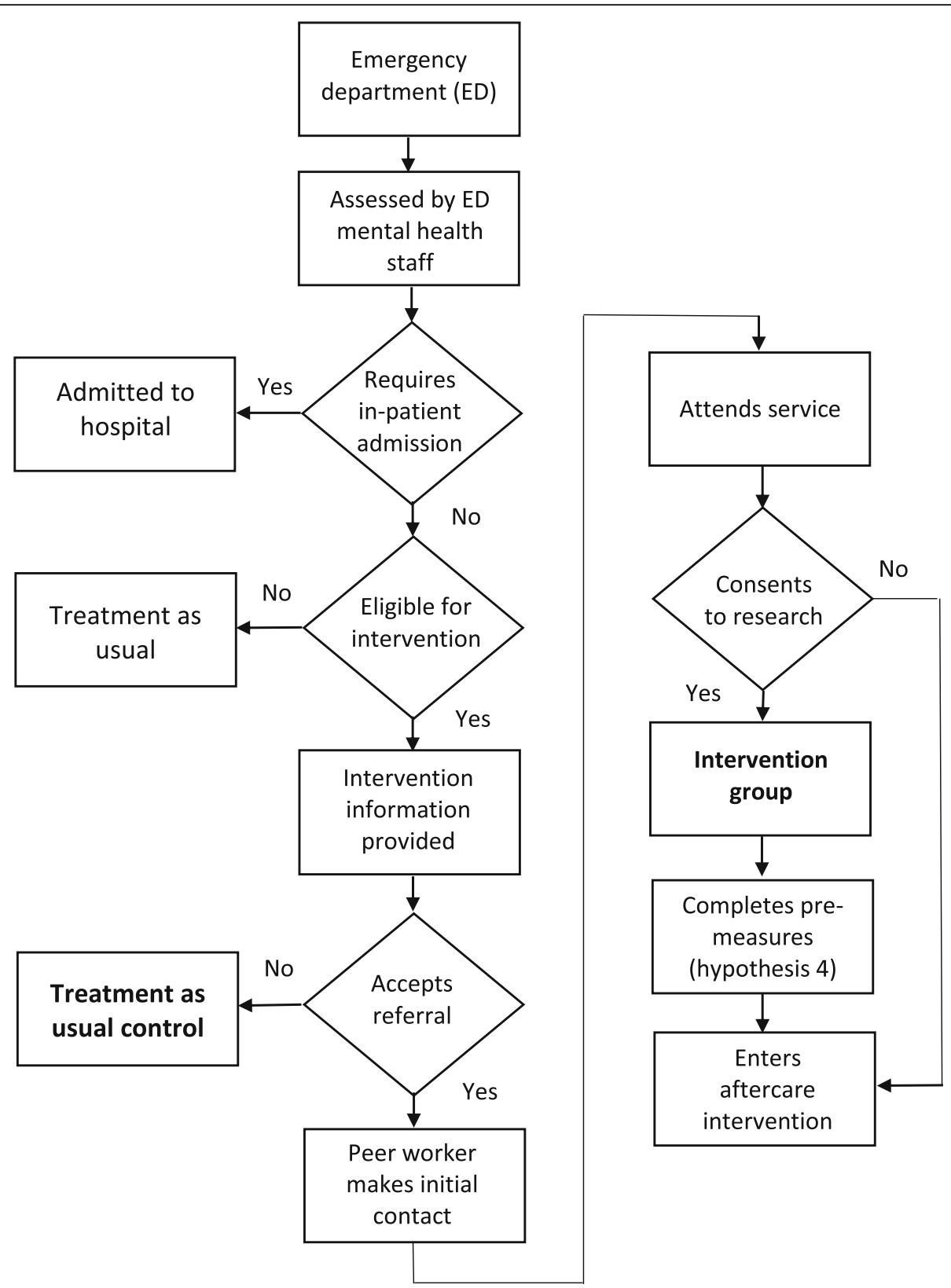

Fig. 1 Participant Recruitment

education on suicide risks and strategies to assist the participant to maintain progress toward his/her goals.

Peer workers will provide follow-up support sessions primarily via telephone (and several face-to-face) three times per week for four weeks; tapering to weekly should the intervention extend to 12 weeks. Each session will be approximately $30 \mathrm{~min}$ in duration. Participants will be coached through the steps in their aftercare plan using the framework of the GROW coaching model. Peer workers are trained in the SafeSide model [71] of risk formulation and will escalate to the mental health clinician should they be concerned about a participant's level of risk.
The clinician and peer worker will meet for discussion and clinical review every two weeks, where they will consider the participant's level of risk and progress, identify any barriers to progression and strategies to address these, and document any linkages to other services the peer worker has facilitated.

The mental health clinician will meet with the participant again at week four, together with the family/carer, if relevant. This face-to-face session will involve review of the aftercare and stabilisation plans, implementation of adjustments if indicated with a view to discharge from the service if appropriate. The participant and clinician 
will conduct the SSF-4 together and discuss any changes that may have occurred on the measures. Participants will be considered suitable for discharge when it is felt that their level of risk is appropriate relative to their level of support and the services with which they are engaged. If the participant is deemed suitable for discharge at this time, a discharge plan will be developed in collaboration with their peer worker and their family/carers. The participant and their family/carer (if relevant) will engage in relapse prevention planning and discharge letters will be sent to the participant's general medical practitioner and any other relevant support services. Prior to discharge, the participant will complete the post-intervention outcome measures. If the participant is not deemed suitable for discharge at their week-four appointment with the clinician, the intervention may extend for up to a further eight weeks. Participants who receive 12 weeks of service and still present with elevated risk will be transitioned to other longer-term options, such as local health district mental health services.

\section{Treatment as usual}

Treatment as usual following a presentation to one of the participating emergency departments for a suiciderelated event involves a mental health Clinical Nurse Consultant recommending, or actively referring, the patient to community-based supports (e.g. general practitioner, counselling, housing support services) and may include follow up by the community mental health team. However, there is no consistent support person to help coordinate these follow ups or check whether the person has in fact connected with any community-based supports. As per local policies, TAU includes one follow-up phone call or home visit by the local health district's mental health outreach team within seven days. A discharge letter is also sent to the patient's nominated general medical practitioner. Other intervention services may already be in place, or arranged as part of these interactions, as required.

\section{Staff training}

\section{Peer workers}

Peer workers are drawn from an established peer worker organisation (Flourish Australia). They undergo a twoday training course in relation to the Aftercare intervention, which provides education regarding the SSF-4, working with aftercare and stabilisation plans, implementing the GROW coaching model, conducting risk assessments and using escalation procedures. Four peer workers are employed by the service.

\section{Mental health clinicians}

Aftercare mental health clinicians are registered practitioners with the Australian Health Practitioner Regulation
Agency. Three clinical psychologists, two registered psychologists and one mental health nurse are employed by the service. All clinicians have attended a four-hour training course regarding the Aftercare intervention, focusing on the development of aftercare and stabilisation plans, the use of the SSF-4, implementation of the GROW coaching model, risk assessments, and procedures for the conduct of clinical review meetings with peer workers. In addition, clinicians have completed a comprehensive online suicide prevention training course, through the Australian Psychological Society, which provides information about suicide and suicidal behaviours, training in risk assessments and crisis management, training in working with populations at serious risk of suicide, information regarding appropriate referral options, and self-care for clinicians.

\section{Treatment Fidelity}

Intervention fidelity will be assessed by measuring treatment adherence using participant's attendance rates for peer worker follow-up and clinician appointments. To maximise intervention integrity, documented procedures will be in place within the Aftercare service to ensure that all workers comply with the intervention protocol. Measures of service indicators, such as adherence to meeting protocols and completion of SSF-4, will be made and audited to provide an assessment of intervention integrity.

\section{Measurements \\ Primary outcomes}

Hospital emergency department data will be extracted to evaluate the primary outcomes of likelihood of a suiciderelated presentation and rate of re-presentations to the three emergency departments as a result of one or more repeat suicide attempt(s) or being at high risk for suicide. Suicide attempt or high risk for suicide will be identified by pre-determined hospital codes. Death registration data will be linked with emergency department data for participants and controls to identify deaths by any cause.

\section{Secondary outcomes}

Secondary outcome measures were chosen as they assess factors associated with increased risk for suicide [64-68]. The measures will be collected from the participants of the Aftercare intervention (pre- and post-) using the following measures.

\section{Hopelessness}

The short form of the Beck Hopelessness Scale (BHSSF) [72] is derived from the original Beck Hopelessness Scale [73] and consists of four True/False items designed to measure hopelessness in the past week. An example of an item is "I might as well give up because I can't 
make things better for myself". The BHS-SF has demonstrated high internal consistency $(\alpha=.85)$ and has been found to be positively correlated with dysfunctional attitudes, exhaustion, psychological distress, hostility, lack of life goals and inability to cope emotionally [72].

\section{Belongingness and burdensomeness}

The 15-item Interpersonal Needs Questionnaire (INQ) utilises a seven-point Likert Scale $(1=$ not at all true for me, 7 = very true for me) designed to measure thwarted belongingness and perceived burdensomeness [74]. An example of an item measuring thwarted belongingness is "These days I am fortunate to have many caring and supportive friends" (reverse scored). An example of an item measuring burdensomeness is "I think my death would be a relief to the people in my life". Both the thwarted belongingness and perceived burdensomeness scales have shown good internal consistency ( $\alpha=.85$ and $\alpha=.89$, respectively) and have been found to demonstrate criterion validity $[74,75]$.

\section{Depression, anxiety and stress}

The 21-item version of the Depression, Anxiety and Stress Scales (DASS-21) [76] measure items on a fourpoint Likert Scale $(0=$ did not apply to me at all, $3=$ applied to me very much or most of the time) designed to gauge symptoms of depression, anxiety and stress. The DASS-21 has an acceptable level of internal consistency with Cronbach's alphas for the three scales ranging from .73 (anxiety) to .81 (depression and stress) [77]. The DASS-21 has been well validated [78-80]. The depression scale of the DASS-21 has been found to predict suicidal ideation [81].

\section{Psychological distress}

The 10-item Kessler Psychological Distress Scale (K-10) [82] consists of 10 items rated on a five-point scale Likert Scale ( $1=$ none of the time, $5=$ all of the time) designed to measure psychological distress over the past four weeks, e.g., "About how often did you feel so nervous that nothing could calm you down?" The K-10 has demonstrated good reliability and validity [83].

\section{Data handling}

Primary outcome data of re-presentations to an emergency department and other local health district data on hospital admissions, community mental health and mental health outcomes will be extracted and de-identified by the Centre for Health Research Illawarra Shoalhaven Population (CHRISP), a research centre which performs data linkage through a partnership between the local health district and the University of Wollongong. Deaths attributable to any cause will be obtained from NSW death registration data held by the NSW Centre for
Health Record Linkage (CHeReL). The researchers with be provided with the de-identified coded data.

Secondary outcome data (suicide risk factors) will be collected by the Aftercare service prior to the participant attending their initial session with a mental health clinician and again at the time of their final (discharge) session with the clinician. For Aftercare participants who consent for their data to be included in the study, the data will be de-identified and coded within the Aftercare service, prior to being provided to the researchers.

Data transfers and storage will comply with national and state legislation, national ethics principles and University of Wollongong, local health district and the CHRISP data management policies.

\section{Data linkage}

The CHRISP data integration officer will link Aftercare and local health district identifiers using deterministic linkage on the Illawarra Health Information Platform. CHeReL will link CHRISP's patient identifier with their own patient identifier using deterministic linkage in the first instance, and probabilistic linkage if required. Probabilistic record linkage allows a calculation of the probability of a match between two or more records to be made when the records are not exactly identical in one or more of the participant identifier fields (e.g., surname 'Browne' or 'Brown'). A record linkage is determined by set thresholds for acceptable probability [84]. Nonidentifiable local health district data will be provided to the researchers by CHRISP. Non-identifiable Aftercare service data will be provided to the researchers by the Aftercare service. Non-identifiable deaths data from the state's Registry of Births, Deaths and Marriages will be provided to the researchers by $\mathrm{CHeReL}$.

\section{Data analysis}

Analyses will be conducted using the Statistical Package for the Social Sciences version 25 [85] and SAS v9.4 [86]. Primary analyses will be conducted according to the intention-to-treat approach. Presentations to an emergency department with a repeat suicide-related event and for all-cause death will be compared between the two non-randomised groups (intervention vs control) using a Kaplan Meier plot where the initial presentation to an emergency department is designated as time zero, and using censoring. A log-rank test will compare the Kaplan Meier curves of the two groups.

A negative binomial regression model will be applied to the data to estimate the effect of the intervention on re-presentation rates, accounting for varying lengths of follow-up period and adjusting for service and personlevel characteristics (e.g. facility, age, sex, indigenous status) and censoring. 
To investigate the impact of the service on suicide risk factors (secondary outcome measures), a comparison of pre- and post-intervention outcome measures will be conducted for participants of the intervention using paired t-tests, or a Wilcoxon signed-ranks test (if the sample is non-normally distributed), adjusting for multiple comparisons (Bonferroni correction).

\section{Sample size and statistical power}

The sample size required to achieve power of $80 \%$ was estimated for the primary analyses using G*Power [87]. Local hospital data (2014/2015 year) indicated that approximately 1340 people present to emergency departments with suicide-related presentations annually and systematic reviews have estimated that $16 \%$ re-attempt suicide within 12 months [5]. Based on Hvid et al.'s [30] report that an intervention group had approximately half the rate of deaths by suicide than a TAU group at 12 months (.087 vs .219), the sample size required for a logrank test at $\alpha$ level .05 is 75 participants in the intervention group and 1265 participants in the control group.

\section{Discussion}

This study aims to contribute to our understanding of effective interventions for people at high-risk for suicide, with the intention of quantifying any impact on the number of presentations to emergency departments for suicide-related events, and all-cause mortality, following an aftercare intervention. The study will provide a realworld examination of an innovative aftercare follow-up service for people who have presented to a hospital emergency department following a suicide attempt or because of high risk for suicide. Specifically, the study will expand upon our understanding of follow up in suicide prevention $[35,88]$, extend literature regarding peer worker integration in treatment models of suicidal persons, and inform future policy decisions.

\section{Limitations}

The study groups are non-randomised, hence intervention participants are self-selecting and may therefore constitute a group that is inherently different from controls prior to study commencement, such as having higher motivation to seek treatment or greater levels of psychological mindedness. Controlling such factors will require future randomised controlled trials. The current study is typical of pragmatic trials which test the effectiveness of interventions in a real-world context as opposed to efficacy trials which are in controlled conditions.

\section{Abbreviations}

BHS-SF: Beck Hopelessness Scale; CAMS: Collaborative Assessment and Management of Suicide; DASS-21: Depression, Anxiety and Stress Scales; GROW: 'Goal, Reality, Options, Wrap-up' coaching model; INQ: Interpersonal
Needs Questionnaire; K-10: Kessler Psychological Distress Scale; NSW: New South Wales; SSF: Suicide Status Form; TAU: treatment-as-usual

\section{Acknowledgements}

We wish to thank the three participating organisations implementing the Aftercare service; Grand Pacific Health, Flourish Australia, and the South Coast Medical Service Aboriginal Corporation. We acknowledge the support of the Illawarra Shoalhaven Local Health District. We would like to thank Professor Frank Deane and Professor Marijka Batterham for advice on research design and statistical analyses. Our appreciation is also expressed to Mr. Tim Heffernan and Mr. Adam McRae for their advice on intervention protocols.

\section{Authors' contributions}

VB had the lead role in study design, conducted literature review and was a major contributor in writing the manuscript. AH contributed to study design and was a major contributor in writing the manuscript. JA conducted literature review and was a major contributor in writing the manuscript. LL provided statistical expertise and contributed to writing the manuscript, particularly the statistical analyses. RS provided advice on intervention protocols and contributed to writing the manuscript. All authors read and approved the final manuscript.

\section{Funding}

VB has received research funding from the University of Wollongong, Faculty of Social Sciences, Research Grant Scheme and the Illawarra Shoalhaven Suicide Prevention Collaborative, Grant Scheme, to assist with this study. These funding bodies play no influencing role in the design or conduct of the research.

\section{Availability of data and materials}

The datasets generated and analysed during the current study are not publicly available due to privacy concerns but are available from the corresponding author on reasonable request and with permission of Grand Pacific Health and the Centre for Health Research Illawarra Shoalhaven Population.

\section{Ethics approval and consent to participate}

The study has been approved by the University of Wollongong Illawarra Shoalhaven Local Health District Human Research Ethics Committee (2017/ 426). Consent to participate in the study will be sought from participants of the Aftercare intervention as they present to the community-based service and prior to their initial appointment with an Aftercare mental health clinician. Participant information sheets will be provided in the waiting room and potential participants will have the opportunity to ask further questions regarding the research from the service staff. They will be advised that they will be free to withdraw from the study at any time, without prejudice. Consent to participate in the study will be verified by the participant's signature on the participant consent form.

Consent for publication

Not applicable.

\section{Competing interests}

$V B, J A$ and $L L$ declare that they have no competing interests. AH played a role in the initial design of the Aftercare intervention. RS is the operational manager of the Aftercare service.

\footnotetext{
Author details

'University of Wollongong, Northfields Avenue, Wollongong, NSW 2522, Australia. ${ }^{2}$ llawarra Health and Medical Research Institute, Northfields Avenue, Wollongong, NSW 2522, Australia. ${ }^{3}$ lllawarra Shoalhaven Suicide Prevention Collaborative, The Central, Innovation Campus, Wollongong, NSW 2500, Australia. ${ }^{4}$ Coordinare, Primary Health Network South Eastern NSW, The Central, Innovation Campus, Squires Way, Wollongong, NSW 2500, Australia. ${ }^{5}$ Centre for Health Research Illawarra Shoalhaven Population, University of Wollongong, Building 234, Innovation Campus, Wollongong, NSW 2500, Australia. ${ }^{6}$ Grand Pacific Health, 336 Keira Street, Wollongong, NSW 2500, Australia.
} 
Received: 3 May 2019 Accepted: 4 October 2019 Published online: 26 October 2019

\section{References}

1. World Health Organization: Suicide Data. 2019. https://www.who.int/ mental_health/prevention/suicide/suicideprevent/en/. Accessed 6 Feb 2019.

2. Australian Bureau of Statistics: Causes of Death, Australia, 2017. http://www. abs.gov.au/ausstats/abs@.nsf/mf/3303.0. Accessed 4 Mar 2019.

3. Geulayov G, Casey D, McDonald KC, Foster P, Pritchard K, Wells C, Clements C, Kapur N, Ness J, Waters K, et al. Incidence of suicide, hospital-presenting non-fatal self-harm, and community-occurring non-fatal self-harm in adolescents in England (the iceberg model of self-harm): a retrospective study. Lancet Psychiatry. 2018:5:167-74.

4. Goldsmith S, Pellmar T, Kleinman A, Bunney WE. Reducing suicide: a national imperative. Washington, DC, US: National Academies Press; 2002.

5. Owens D, Horrocks J, House A. Fatal and non-fatal repetition of self-harm. Systematic review British Journal of Psychiatry. 2002;181:193-9.

6. Benghi M, Rosenbaum JF, Cerri C, Cornaggia CM. Risk factors for fatal and nonfatal repitition of suicide attempts: a literature review. Neuropsychiatr Dis Treat. 2013:9:1725-36.

7. Hunt IM, Kapur N, Webb R, Robinson J, Burns J, Shaw J, Appleby L. Suicide in recently discharged psychiatric patients: a case-control study. Psychol Med. 2009;39:443-9.

8. Haw C, Bergen H, Casey D, Hawton K. Repetition of deliberate self-harm: a study of the characteristics and subsequent deaths in patients presenting to a general hospital according to extent of repetition. Suicide Life Threat Behav. 2007:37:379-96.

9. Shand F, Christensen H, Pirkis J, Batterham P, Spittal M, Woodward A, Buckley H, McKay K, Tighe J. Care after a suicide attempt. Hobart, Australia: In: National Suicide Prevention Conference.; 2015.

10. Olfson M, Marcus SC, Bridge JA. Emergency treatment of deliberate selfharm. Arch Gen Psychiatry. 2012;69:80-8.

11. Cooper J, Steeg S, Bennewith O, Lowe M, Gunnell D, House A, Hawton K, Kapur N. Are hospital services for self-harm getting better? An observational study examining management, service provision and temporal trends in England. BMJ Open. 2013;3:-e003444.

12. Spittal MJ, Shand F, Christensen H, Brophy L, Pirkis J. Community mental health care after self-harm: a retrospective cohort study. Australian \& New Zealand Journal of Psychiatry. 2016;51:727-35.

13. Shand FL, Batterham PJ, Chan JKY, Pirkis J, Spittal MJ, Woodward A, Christensen $\mathrm{H}$. Experience of health care services after a suicide attempt: results from an online survey. Suicide Life Threat Behav. 2018;48:779-87.

14. Black Dog Institute: Improving emergency and follow-up care for suicidal crisis. https://blackdoginstitute.org.au/research/lifespan/lifespan-strategiesand-components/strategy-1 (2018). Accesssed 9 Nov 2018.

15. Mann JJ, Apter A, Bertolote J, Beautrais A, Currier D, Haas A, Hegerl U, Lonnqvist J, Malone K, Marusic A, et al. Suicide prevention strategies: a systematic review. JAMA. 2005;294:2064-74.

16. van Walraven C, Mamdani M, Fang J, Austin PC. Continuity of care and patient outcomes after hospital discharge. J Gen Intern Med. 2004;19:624-31.

17. Kripalani S, LeFevre F, Phillips CO, Williams MV, Basaviah P, Baker DW. Deficits in communication and information transfer between hospital-based and primary care physicians: implications for patient safety and continuity of care. JAMA. 2007;297:831-41.

18. Phillips CO, Wright SM, Kern DE, Singa RM, Shepperd S, Rubin HR. Comprehensive discharge planning with Postdischarge support for older patients with congestive heart FailureA meta-analysis. JAMA. 2004;291:1358-67.

19. Naylor MD, Brooten D, Campbell R, Jacobsen BS, Mezey MD, Pauly MV, Schwartz JS. Comprehensive discharge planning and home follow-up of hospitalized elders: a randomized clinical trial. JAMA. 1999;281:613-20.

20. Brooten D, Kumar $S$, Brown LP, Butts P, Finkler SA, Bakewell-Sachs $S$, Gibbons A, Delivoria-Papadopoulos M. A randomized clinical trial of early hospital discharge and home follow-up of very-low-birth-weight infants. N Engl J Med. 1986;315:934-9.

21. Brooten D, Roncoli M, Finkler S, Arnold L, Cohen A, Mennuti M. A randomized trial of early hospital discharge and home follow-up of women having cesarean birth. Obstet Gynecol. 1994;84:832-8.

22. Sanddal ND, Sanddal TL, Berman AL, Silverman MM. A general systems approach to suicide prevention: lessons from cardiac prevention and control. Suicide Life Threat Behav. 2003;33:341-52.
23. Baker STE, Nicholas J, Shand F, Green R, Christensen H. A comparison of multi-component systems approaches to suicide prevention. Australasian Psychiatry. 2017;26:128-31.

24. National Action Alliance for Suicide Prevention. Suicide care in systems framework. In: National Action Alliance for Suicide Prevention, 2019. https:// theactionalliance.org/sites/default/files/clinicalcareinterventionreport.pdf (2011). Accessed 8 Feb 2019

25. van der Feltz-Cornelis CM, Sarchiapone M, Postuvan V, Volker D, Roskar S, Grum AT, Carli V, McDaid D, O'Connor R, Maxwell M, et al. Best practice elements of multilevel suicide prevention strategies: a review of systematic reviews. Crisis. 2011;32:319-33.

26. Ridani R, Torok M, Shand F, Holland C, Murray S, Borrowdale K, Sheedy M, Crowe J, Cockayne N, Christensen H. An evidence-based systems approach to suicide prevention: guidance on planning, commissioning, and monitoring. Black Dog Institute: Sydney, Australia; 2016.

27. Krysinska K, Batterham PJ, Tye M, Shand F, Calear AL, Cockayne N, Christensen $\mathrm{H}$. Best strategies for reducing the suicide rate in Australia. Australian \& New Zealand Journal of Psychiatry. 2015;50:115-8.

28. Dieserud G, Loeb M, Ekeberg O. Suicidal behavior in the municipality of Bærum, Norway: a 12-year prospective study of Parasuicide and suicide. Suicide Life Threat Behav. 2000;30:61-73.

29. Hvid M, Wang AG. Preventing repetition of attempted suicide-I. feasibility (acceptability, adherence, and effectiveness) of a Baerum-model like aftercare. Nordic Journal of Psychiatry. 2009;63:148-53.

30. Hvid M, Vangborg K, Sorensen HJ, Nielsen IK, Stenborg JM, Wang AG. Preventing repetition of attempted suicide--II. The Amager project, a randomized controlled trial. Nordic Journal of Psychiatry. 2011;65:292-8.

31. Lahoz T, Hvid M, Wang AG. Preventing repetition of attempted suicide-III. The Amager project, 5-year follow-up of a randomized controlled trial. Nordic Journal of Psychiatry. 2016;70:547-53.

32. Milner AJ, Carter G, Pirkis J, Robinson J, Spittal MJ. Letters, green cards, telephone calls and postcards: systematic and meta-analytic review of brief contact interventions for reducing self-harm, suicide attempts and suicide. Br J Psychiatry. 2015;206:184-90.

33. Miller IW, Camargo CA Jr, Arias SA, Sullivan AF, Allen MH, Goldstein AB, Manton AP, Espinola JA, Jones R, Hasegawa $K$, et al. Suicide prevention in an emergency department population: the ED-SAFE study. JAMA Psychiatry. 2017:74:563-70.

34. Kaminer Y, Burleson JA, Goldston DB, Burke RH. Suicidal ideation among adolescents with alcohol use disorders during treatment and aftercare. Am J Addict. 2006;15(Suppl 1):43-9.

35. Fleischmann A, Bertolote JM, Wasserman D, De Leo D, Bolhari J, Botega NJ, De Silva D, Phillips M, Vijayakumar L, Varnik A, et al. Effectiveness of brief intervention and contact for suicide attempters: a randomized controlled trial in five countries. Bull World Health Organ. 2008;86:703-9.

36. Motto JA, Bostrom AG. A randomized controlled trial of Postcrisis suicide prevention. Psychiatr Serv. 2001;52:828-33.

37. Carter GL, Clover K, Whyte IM, Dawson AH, Este CD. Postcards from the EDge project: randomised controlled trial of an intervention using postcards to reduce repetition of hospital treated deliberate self poisoning. BMJ. 2005; 331:805.

38. Carter GL, Clover K, Whyte IM, Dawson AH, D'Este C. Postcards from the EDge: 24-month outcomes of a randomised controlled trial for hospitaltreated self-poisoning. Br J Psychiatry. 2007;191:548-53.

39. Carter GL, Clover K, Whyte IM, Dawson AH, D'Este C. Postcards from the EDge: 5-year outcomes of a randomised controlled trial for hospital-treated self-poisoning. Br J Psychiatry. 2013;202:372-80.

40. Bilén K, Pettersson H, Owe-Larsson B, Ekdahl K, Ottosson C, Castrén M, Ponzer S. Can early follow-up after deliberate self-harm reduce repetition? A prospective study of 325 patients. J Affect Disord. 2014;152-154:320-5.

41. Brown GK, Green KL. A review of evidence-based follow-up Care for Suicide Prevention: where do we go from Here? Am J Prev Med. 2014;47(Suppl 2): 209-15

42. Peer Work Hub. Employer's guide to implementing a peer workforce - A case for your organisation. Peer Workforce Hub. 2019. http://peerworkhub. com.au/wp-content/uploads/2016/05/Business-Case.pdf (2016). Accessed 8 Feb 2019.

43. Davidson L, Chinman M, Sells D, Rowe M. Peer support among adults with serious mental illness: a report from the field. Schizophr Bull. 2006;32:443-50.

44. Clarke GN, Herinckx HA, Kinney RF, Paulson RI, Cutler DL, Lewis K, Oxman E. Psychiatric hospitalizations, arrests, emergency room visits, and 
homelessness of clients with serious and persistent mental illness: findings from a randomized trial of two ACT programs vs. usual care. Ment Health Serv Res. 2000;2:155-64.

45. Min SY, Whitecraft J, Rothbard AB, Salzer MS. Peer support for persons with co-occurring disorders and community tenure: a survival analysis. Psychiatric Rehabilitation Journal. 2007:30:207-13.

46. Gillard S, Holley J. Peer workers in mental health services: literature overview. Adv Psychiatr Treat. 2014;20:286-92.

47. Pitt V, Lowe D, Hill S, Prictor M, Hetrick SE, Ryan R, Berends L. Consumerproviders of care for adult clients of statutory mental health services. Cochrane Database Syst Rev. 2013. https://doi.org/10.1002/14651858.

48. Passmore J, editor. Excellence in coaching: the industry guide. London: Kogan Page; 2006

49. Alexander G, Renshaw B. Super-coaching. London: Random House; 2005

50. Whitmore J. Coaching for performance: growing people, performance and purpose. London: Nicholas Brealey; 2002.

51. Deane FP, Crowe TP, Oades LG, Ciarrochi J, Marshall S, Williams V, Andresen R. Facilitating the transfer of collaborative recovery training into clinical practice: intervention and coaching protocols. Illawarra Insitute for Mental Health, Unviersity of Wollongong: Wollongong, Australia; 2010.

52. Hetrick SE, Robinson J, Spittal MJ, Carter G. Effective psychological and psychosocial approaches to reduce repetition of self-harm: a systematic review, meta-analysis and meta-regression. BMJ Open. 2016;6:-e011024.

53. Jobes DA. Managing suicidal risk: a collaborative approach (2nd edition). New York: The Guilford Press; 2016.

54. Jobes DA, Jacoby AM, Cimbolic P, Hustead LAT. Assessment and treatment of suicidal clients in a university counseling center. Couns Psychol. 1997;44: 368-77.

55. Jobes DA, Kahn-Greene E, Greene JA, Goeke-Morey M. Clinical improvements of suicidal outpatients: examining suicide status form responses as predictors and moderators. Archives of Suicide Research: Official Journal of the International Academy for Suicide Research. 2009;13: 147-59.

56. Jobes DA, Wong SA, Conrad AK, Drozd JF, Neal-Walden T. The collaborative assessment and management of suicidality versus treatment as usual: a retrospective study with suicidal outpatients. Suicide \& Life-threatening Behavior. 2005;35:483-97.

57. Arkov K, Rosenbaum B, Christiansen L, Jonsson H, Munchow M. Treatment of suicidal patients: the collaborative assessment and Management of Suicidality. Ugeskr Laeger. 2008;170:149-53.

58. Nielsen AC, Alberdi F, Rosenbaum B. Collaborative assessment and management of suicidality method shows effect. Dan Med Bull. 2011;58:A4300.

59. Ellis TE, Green KL, Allen JG, Jobes DA, Nadorff MR. Collaborative assessment and management of suicidality in an inpatient setting: results of a pilot study. Psychotherapy. 2012;49:72-80.

60. Ellis TE, Rufino KA, Allen JG, Fowler JC, Jobes DA. Impact of a suicidespecific intervention within inpatient psychiatric care: the collaborative assessment and Management of Suicidality. Suicide \& Life-threatening Behavior. 2015;45:556-66

61. Ellis TE, Rufino KA, Allen JG. A controlled comparison trial of the collaborative assessment and Management of Suicidality (CAMS) in an inpatient setting: outcomes at discharge and six-month follow-up. Psychiatry Res. 2017;249:252-60.

62. Marshall E, York J, Magruder K, Yeager D, Knapp R, De Santis ML, Burriss L, Mauldin M, Sulkowski S, Pope C, et al. Implementation of online suicidespecific training for VA providers. Academic Psychiatry: The Journal of the American Association of Directors of Psychiatric Residency Training and the Association for Academic Psychiatry. 2014;38:566-74.

63. Crowley KJ, Arnkoff DB, Glass CR, Jobes DA. Collaborative assessment and management of suicidality: perspectives from the Catholic University suicide prevention lab. Los Angeles, CA: In: Annual conference of the American Association of Suicidology; 2014.

64. Too LS, Spittal MJ, Bugeja L, Reifels L, Butterworth P, Pirkis J. The association between mental disorders and suicide: a systematic review and metaanalysis of record linkage studies. J Affect Disord. 2019;259:302-13.

65. Wu C, Chen Z, Yu L, Duan W, Jiang G. Effects of depression and hopelessness on suicide ideation: the mediation effect of psychache. Chinese Journal of Clinical Psychology. 2015;23:1040-3.

66. Naidoo S, Collins S. A test of the interpersonal-psychological theory of suicidal behaviour in a sample of mental health outpatients. S Afr J Psychol. 2019:49:430-45.
67. Batty GD, Kivimaki M, Bell S, Gale CR, Shipley M, Whitley E, Gunnell D. Psychosocial characteristics as potential predictors of suicide in adults. An overview of the evidence with new results from prospective cohort studies Translational Psychiatry. 2018;8:00728.

68. Tanji F, Tomata Y, Zhang S, Otsuka T, Tsuji I. Psychological distress and completed suicide in Japan: a comparison of the impact of moderate and severe psychological distress. Prev Med. 2018;116:99-103.

69. NSW Government: Health Stats. http://www.healthstats.nsw.gov.au (2018). Accessed 8 Nov 2018.

70. Gosh a. suicide update snapshot - Nov 2017. In: COORDINARE - South Eastern NSW Primary Health Network Report 2017.

71. Pisani T: SafeSide Prevention. https://www.safesideprevention.com (2018) Accessed 4 March 2019

72. Perczel Forintos D, Rózsa S, Pilling J, Kopp M. Proposal for a short version of the Beck hopelessness scale based on a National Representative Survey in Hungary. Community Ment Health J. 2013;49:822-30.

73. Beck AT, Weissman A, Lester D, Trexler L. The measurement of pessimism: the hopelessness scale. J Consult Clin Psychol. 1974;42:861-5.

74. Van Orden KA, Witte TK, Gordon KH, Bender TW, Joiner TE Jr. Suicidal desire and the capability for suicide: tests of the interpersonal-psychological theory of suicidal behavior among adults. J Consult Clin Psychol. 2008;76: 72-83.

75. Van Orden KA, Cukrowicz KC, Witte TK, Joiner TE. Thwarted belongingness and perceived burdensomeness: construct validity and psychometric properties of the interpersonal needs questionnaire. Psychol Assess. 2012;24: $197-215$.

76. Lovibond SHL, P. F. Manual for the depression anxiety stress scales (2nd edition). In. Sydney, Australia: Psychology Foundation; 1995.

77. Lovibond PF, Lovibond SH. The structure of negative emotional states: comparison of the depression anxiety stress scales (DASS) with the Beck depression and anxiety inventories. Behav Res Ther. 1995;33:335-43.

78. Henry JD, Crawford JR. The 21 item version of the depression anxiety stress scales (DASS-21): normative data and psychometric evaluation in a large non-clinical sample. Br J Clin Psychol. 2005;44:227-39.

79. Clara IP, Cox BJ, Enns MW. Confirmatory factor analysis of the depressionanxiety-stress scales in depressed and anxious patients. J Psychopathol Behav Assess. 2001;23:61-7.

80. Antony MMB, Bieling PJ, Cox Brian J, Enns MW, Swinson RP. Psychometric properties of the 42 -item and 21-item versions of the depression anxiety stress scales in clinical groups and a community sample. Psychol Assess. 1998;10:176-81.

81. Ibrahim N, Amit N, Suen MWY. Psychological factors as predictors of suicidal ideation among adolescents in Malaysia. PLoS One. 2014. https://doi.org/10. 1371/journal.pone.

82. Kessler RC, Andrew G, Colpe LJ. Short screening scales to monitor population prevalences and trends in non-specific psychological distress. Psychol Med. 2002;32:959-76.

83. Hides L, Lubman DI, Devlin H, Cotton S, Aitken C, Gibbie T, Hellard M. Reliability and validity of the Kessler 10 and patient health questionnaire among injecting drug users. Australian \& New Zealand Journal of Psychiatry. 2007:41:166-8.

84. Sayer A, Ben-Shlomo Y, Blom AW, Steele F. Probabilistic record linkage. Int J Epidemiol. 2016;45:954-64

85. Corp IBM. IBM SPSS statistics for windows, version 25.0. Armonk: IBM Corp; 2017.

86. SAS Institute. SAS Version 9.4. SAS Institute Inc: Cary NC.

87. Faul F, Erdfelder E, Buchner A, Lang A-G. Statistical power analyses using G*power 3.1: tests for correlation and regression analyses. Behav Res Methods. 2009:41:1149-60.

88. Zalsman $\mathrm{G}$, Hawton $\mathrm{K}$, Wasserman $\mathrm{D}$, van Heeringen $\mathrm{K}$, Arensman $\mathrm{E}$, Sarchiapone M, Carli V, Hoschl C, Barzilay R, Balazs J, et al. Suicide prevention strategies revisited: 10-year systematic review. Lancet Psychiatry. 2016;3:646-59.

\section{Publisher's Note}

Springer Nature remains neutral with regard to jurisdictional claims in published maps and institutional affiliations. 